\title{
58 \\ VIRSIM: a methodology supported by an integrated software for the Re-engineering of the factories
}

J BORDA ELJABARRIETA,

A. POLO

DA TALDE SISTEPLANT, S.A. and Deusto University

Parque Tecnologico Edificio 10548170 ZAMUDIO SPAIN

Phone.- (34) 44520611 Fax: (34) 44521266

E-mail.-datalde@redestb.es

R. JOCHEM,

Fraunhofer Institute of production Systems and Design

Technology (IPK) Berlin

Pascaslstrasse 8-9, D- 10587 BERLIN GER@NY

Phone.- (49) 3039006195 Fax.- (49) 303932503

E-mail.-rolandjochem@ipkjhg.de

G. DOUMEINGTS, A. MOUDDEN

LAPIGRAI, Universiti Bordeaux 1

351 Cours de la Libiration, 33405 TALENCE France

Phone (33) 556846530 Fax.- (33) 556846644

E-mail.-doum@lap.u-bordeauxft 


\begin{abstract}
Frequent plant lay-out and process automation changes is becoming more and more critical for production efficiency and reliability in companies with self developed or induced product design changes.

The paper presents VIRSIM (Vlrtual reality and SIMulation), a methodology supporting the complete reengineering process based on GIM (GRAI Integrated Methodology) and IEM (Integrated Enterprise Modeling), and the associated software tool consisting of five subsystems, some of them commercial available low cost software packages. Potential interested industries are automotive components-makers and those with large production engineering departments in general.
\end{abstract}

Keywords

Virtual reality simulation, layout optimization, GIM, IEM.

\title{
1. INTRODUCTION
}

A yearly layout change? The continuous evolution of business oblige the manufacturing companies to re-engineer processes and to move machines to optimize the flow of products through the workshops: these tasks oblige to redesign, to implement solutions to train people. These changes are performed generally in a very short time, using some periods in which the company decreases its business (usually summer in Europe).

A methodology for re-engineering factories and an integrated software system supporting the enterprise modeling and design for both the physical process and the organizational aspects at the conceptual level, that represents in virtual reality physical systems and that simulates correspondent management processes (order processing, planning, scheduling, dispatching, etc.), giving also practical output design issues such detailed drawings, MTM data, specifications, for automation etc. is going to be decisive in interactive design and Virtual Reality trial and error and training, thus saving more than $70 \%$ of the lead-time needed in the complete process from idea to implementation. Another critical point is that will appear a remaining time immediately useful in two critical aspects: concentrating in preventive actions to avoid delicate adjustments in the machines with the move, and also in designing very well fitted man/machine interfaces strategy. 


\section{THE VIRSIM METHODOLOGY}

The VIRSIM Methodology combines the strengths of the GRAI approach (Doumeingts 84, Zanettin 94) and IEM (Mertins, 92). It uses the GRAI approach for the modeling and design of the decisional part of manufacturing systems and IEM for the modeling and design of the physical system. Before giving the main principles of the VIRSIM methodology, we describe briefly the key concepts of the GRAT approach and IEM.

\subsection{The GRAI Approach}

Four different formalisms are used in the modeling of manufacturing systems with the GRAI approach: (GRAI grids and nets, IDEFO and Entity/Relationship diagrams). However, in order to simplify the presentation, we will focus solely on the decisional system and its representation through the GRAI grid. The grid has a matrix form which is used to detail temporal and functional aspects of the decisional system, as shown in Figure 1.

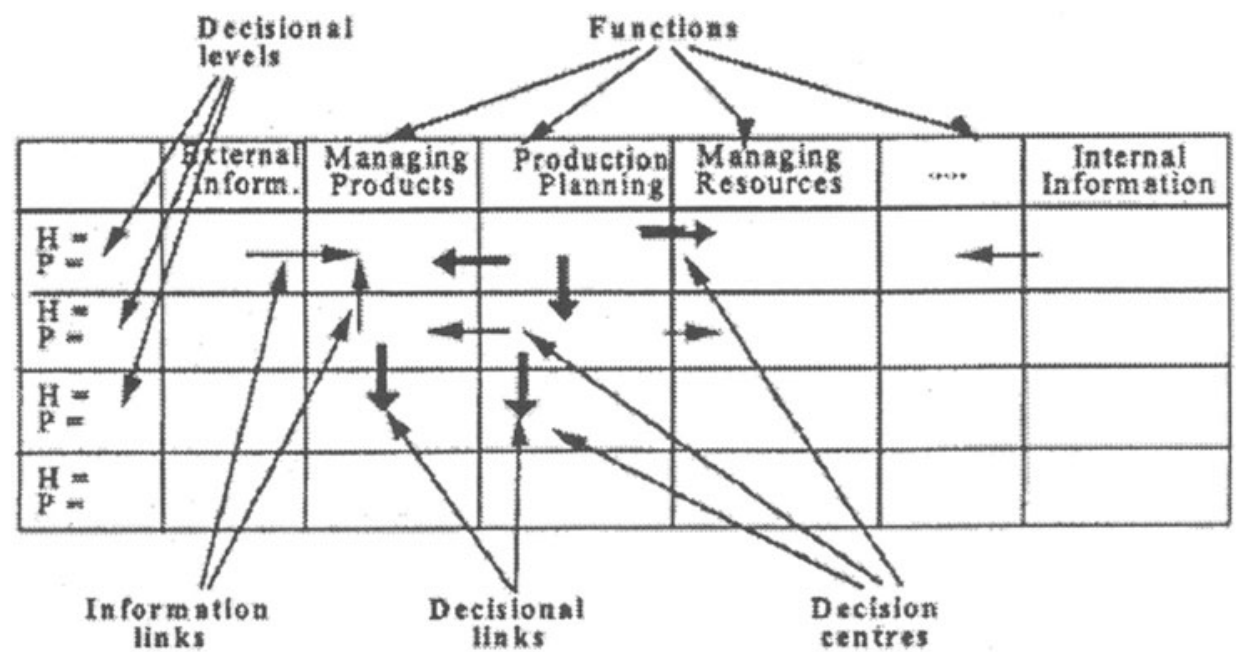

Figure l: GRAI grids formalism.

The following components can be identified in the grid: 
- the functions, describing the set of decision activities that are performed in order to achieve a particular goal. In Figure I we show the three basic functions of manufacturing systems: Managing Products, Production Planning and Managing Resources.

- the decisional levels, defined in terms of Horizon/Period pairs. The Horizon corresponds to an interval, on which a decision is valid, while the Period corresponds to a time interval when the decisions established in the Horizon are reconsidered.

- the decision centers, represented in the slots of the grid, group together all the activities belonging to the same function and the same decision level.

- the information links, determining the information flow from one decision center to another.

- the decisional links, determining the information items which is necessary for a decision to be taken.

- the external information, representing external data which serves as an interface between the system studied and its environment. The internal information, in contrast, represents the data that is created within the manufacturing system.

The grid, as well as the other GRAI formalisms, are used in the first phase of the GRAI approach (the modeling phase) to model the existing manufacturing system, giving an emphasis on the decisional subsystem. The two subsequent phases of the approach are: the inconsistency search phase, whose main objective is to detect weak points in the system studied; and the design phase, where the design of the new manufacturing system is elaborated based on the results of the previous phase.

Throughout a GRAI study', several groups of people are formed. Among them, two have a very important role:

- the synthesis group, composed of managers and other members of staff who are responsible for the manufacturing functions considered. The synthesis group is in charge of validating the results obtained in the different phases of a GRAI study.

- the analysis group, composed of one or more analysts (according to the work load), and one GRAI specialist. The role of the analysis group is to collect the relevant information for the study, to analyze it and to propose solutions for the problems detected.

\subsection{The IEM methodology}

In order to provide a comprehensive and extendible enterprise model, the IEM method uses the object-oriented modeling approach, thus allowing the integration of different views on an enterprise in one consistent model as well as the easy adaptation of the model to changes within the enterprise. 
Generic classes of objects

The generic classes 'Product', 'Resource' and 'Order' are the basis of Integrated Enterprise Modeling for developing models from a user's point of view. They will be specified according to the specifications of an individual enterprise. Each generic class prescribes a specific generic attribute structure, thus defining a frame for describing the structure and behavior of objects of its subclasses. Real enterprise objects will be modeled as objects of these subclasses.

Required enterprise data and the business processes, i.e. the tasks referring to objects, be structured in accordance with the object classes. Furthermore, the relations between objects are determined. The result is a complete description of tasks, business processes, enterprise data, production equipment and information systems of the enterprise at any level of detail.

The model core comprises two main views. The tasks, which are to be executed on objects, and the business processes are the focal point of the Process Model View, whereas the Information Model View primarily regards the objects describing data. Therefore, the core of the enterprise model consists of the data and process representation of classes of objects. The views are interlinked by referring to the same objects and activities, although they represent them in different ways, levels of detail and context. Any view on the model can be derived from this standardized model core. Additional features may be attaches to the core if necessary.

\section{Business Processes as Interactions of Objects}

Everything that happens in a manufacturing enterprise as part of the manufacturing process can be described by activities. In general, activities process and modify objects which were classified above as 'Products', 'Orders' and 'Resources'. The execution of any activity requires direct or indirect planning and scheduling. It is executed by resources which posses the required capacities. The IEM method suggests three levels when describing the essentials of an activity.

- The Action is an object-independent description of any task or business, a verbal description of some task, process step or procedure.

- The Function describes the processing of objects as a transformation from one determined (beginning) state to another determined (ending) state.

- The Activity specifies the order, which controls the execution of the function, and the resource(s), which is (are) in charge of executing the function.

Figure 2 graphically represents the Generic Activity Model. The beginning and ending states are connected with the action rectangle by arrows from left to right. The controlling of the activity is represented by an order state description and a dashed vertical arrow from the top; the required or actually assigned capability for 
executing the function is represented by a resource state description and a dashed vertical arrow from the bottom.

' $A$ GRAI study is the term used to refer to a complete enterprise modeling case using the GRAI approach.

Product or Onder or Resourte objects resp. to be processed

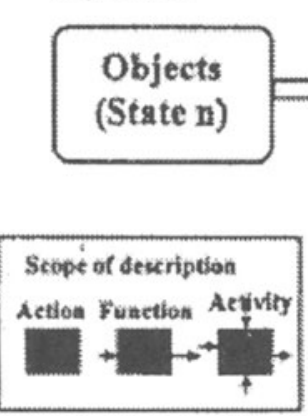

Orifor

Conircis the object processing

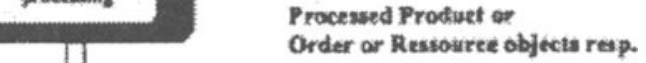

Figure 2: Interactions of Objects Described by the Generic Activity Model.

The Generic Activity Model represents the processing of objects of the product, order or resource class respectively indicating the interactions of objects while processing. The related organizational structure is described by specific resource classes along with their interrelations.

Using special linking constructs, actions, functions and activities are combined to represent business processes. The decomposition and aggregation of processes is also supported. 
Unlike the GRAI approach and IEM, the VIRSIM methodology intends to support the process of plants reengineering beginning from enterprise modeling and finishing with the implementation of the new optimized plant.

For enterprise modeling, VIRSIM uses the formalisms of IEM (the Process Model using the Generic Activity Model) for representing the physical system and the formalisms of the GRAI approach (GRAI grids and nets) to model the decisional system. IEM and the GRAI approach are integrated trough the concepts of 'Product' and 'Resource'. As we can see in the grid in Figure 1, the two functions 'Managing Products' and 'Managing Resources' use as subject the objects derived or instanciated from the IEM generic classes 'Product' and 'Resources'.

The different steps of the VIRSIM methodology approach are given below:

1. Model the existing physical system using IEM formalisms (Process Model)

2. Model the existing decisional system (driving the physical system) using the GRAI formalisms (GRAI grids and nets)

3. Evaluate and optimize the models by determining the weak points and by correcting them

4. Use the optimized physical system model to generate a rough layout of the future plant and transmit it to a CAD system

5. Enhance the rough layout using the CAD system and transmit it to a simulator

6. Simulate global logistics and get the logistic optimum model and the transfer lot

7. Automatically adjust the CAD layout

8. Design and simulate cell and work-place systems by means of Virtual Reality and obtain MTM optimum processes

9. Perform the 6 and 8 steps until proper results are obtained

10. Automation and $\mathrm{man} /$ machine interfacing

11. Perform step 7

12. Adapt the initial models if necessary

To do this last issue, it is necessary to characterize the physical layout, and this is done in tens of two items extracted from the logistic simulator (see Figure 3, system 5):

- Production strategy: MTS (Manufacturing to stock), ATO (assemble to order), MTO (manufacturing to order)

- Related main and auxiliary lines:

The main line is synchronized, time buffered inventory, contains the process technology, and sets the manufacturing lead-times. Main line ( differentiation on 
product inventory, quality, flexibility and service, auxiliary lines (machines and subcontractors) are just opposite decoupled systems.

\section{THE VIRSIM TOOL}

In order to support the VIRSIM methodology, a software tool is being developed. The architecture of this tool is given in Figure 3. It is composed of five subsystems and six interfaces between these subsystems.

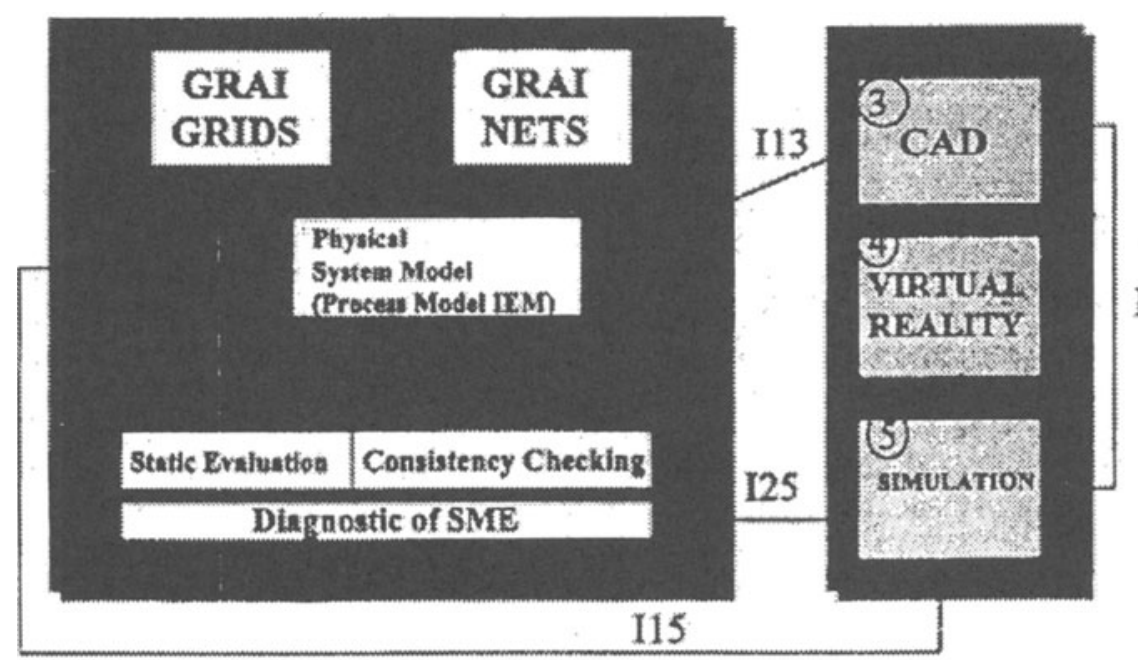

Figure 3: The architecture of the VIRSIM tool.

System 1: $\quad$ Integrated VIRSIM graphical editors.

Function: Enterprise Modeling

System 2: $\quad$ Static Evaluation, Consistency Checking and Diagnostic of the SME system

Function: Static evaluation of the physical system model against selected criteria (rough costs and lead-time). Consistency Checking of the models (at the syntactical level). Rule-based diagnostic of the SME system (semantic check).

System 3: $\quad$ 2D/3D low cost standard CAD. 
Function: I/O layout dimensions

System 4: $\quad$ Low cost Virtual Reality (VR) software.

Function: $\quad$ Solid representation of Icons .

Workplace ergonomics and interference's.

System 5.- $\quad$ Simulation software.

Function: Material handling optimization, queuing and line balancing.

The interfaces description is given below:

a) Interface 134

Use of the layout obtained through $\mathrm{CAD}$ as the background of the simulator.

The figures (dimensions) drawn in $\mathrm{CAD}$ are integrated in the mathematics of the simulator.

b) Interface $\mathrm{I}_{35}$

Double clicking on a particular area or system either of the $\mathrm{CAD}$ drawing or the simulator will allow zooming a 3D solid representation in the VR system.

The 2D dimensions displayed in the CAD system or the ones optimized by the simulator (i.e.: buffer sizing) can be used as restrictions to build the $3 \mathrm{D}$ solid model of the VR system.

$3 \mathrm{D}$ dimensions from the VR are imported to automatically build up a CAD $3 D$ drawing.

c) Interface $\mathrm{I}_{45}$

Double clicking on the simulator allows zooming a 3D solid representation in the VR system.

2D dimensions of the layout displayed on the simulator can be used as restrictions to build the 3D solid model of the VR system.

Coherence between cycle times deduced from the simulator and the workplace MTM analysis obtained by the VR system.

d) Interface $\mathrm{I}_{13}$

The model of the physical system obtained using the editors (System 1) and the VIRSIM methodology is used as input by the CAD system (system 3). Using the data contained in this model, the interface generates automatically a rough drawing.

e) Interface $\mathrm{I}_{25}$

Through this interface, the rule-based diagnostic system will analyze the results of the simulation and will present the results to the user. In addition, the simulation 
tools will receive specific constraints from the diagnostic module (to focus the simulation on a particular area, for instance, to point out bottlenecks).

f) Interface $\mathrm{I}_{15}$

The results of the simulation will lead, if they are not satisfactory, to the modification of the physical model, the whole process will have to be repeated.

\section{CONCLUSIONS}

We have presented in this paper the main principles of a new methodology dedicated to complete frequent factory re-engineering. In addition, we described the associated software tool, giving the details of its different modules and interfaces.

The VIRSIM methodology and its supporting tool are presently under development by the R\&D Department of DATALDE SISTEPLANT, S.A. in consortium with the LAP/GRAI, the division of Systems Planning of the Fraunhofer IPK Berlin, INTEGRAI and several leading car components-makers in Spain.

\section{REFERENCES}

Doumeingts, G., (I 984). Methode GRAI, methods de conception des syst@mes en productique. Doctoral Thesis, Universitd Bordeaux 1.

Zanettin, M., (1994). ContributionLi une mgthode de conception de systems de production. PhD Thesis, Universit6 Bordeaux 1.

Mertins, K., Jochem, R (1992) An Object Oriented Method for Integrated Enterprise modeling as a basis for Enterprise Coordination. International Conference on Enterprise Integration Modeling Technolo8y (ICEIMT). Hilton Head (South Carolina), US Air Force-Integration Technology Division.

\section{BIOGRAPHIES}

ProL Dr. Javier Borda Elejabarietta has been working as plant Engineer and Production Manager staff for 7 years, and from 1984 he is the managing director and C.E.O. of DATALDE, S.A., a Spanish 35 people industrial engineering company, sited in the Basque Country. He read in 1989 the Doctoral Mechanical Engineering dissertation on "CIM for plastic injection workshops". He is also Professor of Production and Engineerincr Management in the University of 
Deusto, Bilbao, and the author of several international papers and a book titled: "Advanced Maintenance Techniques" (1990). He has become recently an IFIP WG 5.7 member.

Alvaro Polo is a Mechanical Engineer at DATALDE SISTEPLANT, S.A. Bilbao Spain.

Dipi.-Ing. Roland Jochem has obtained his MS in 1987 in Mechanical Engineering at the Technical University of Berlin. He has been working as Mechanical engineer at a company for special machines in Berlin from 1987 to 1988. Since 1989, he is a Scientist at the Fraunhofer-Institute for Production System and Design Technology (IPK) and since 1992 he is the responsible for the research group Business Process Modeling. His is an expert on business planning, specialized on production integration strategies, business process modeling, and information modeling and modeling methods. Roland Jochem is a member of ISO TC 184 SC5/WGI (Modeling and Architecture) and CEN/CENELEC/WG-ARC (Architecture and Enterprise Modeling).

Guy Doumeingts is Professor at the University Bordeaux 1. He obtained his $\mathrm{PhD}$ degree in 1967, from this same institution. He participated in the setting-up of the GRAI research group from the LAP, of which he is the Director since 1989. He has held many research contracts, published two books, and over 200 papers on CIM. He is expert for the Commission of the European Communities (ESPRIT Project), French representative to IFIP,

Chairman of the Technical Committee 45 (Computer Application in Technology), and member of the editorial board of several international journals.

Adnane Moudden is a research Engineer at the GRAI research group belonging to the LAP (Laboratoire d'Automatique et de Productique). He obtained his $\mathrm{PhD}$ degree from the University Bordeaux I in 1997. His research interests include case-based reasoning, neural-fuzzy systems, machine learning and the application of these fields to enterprise modeling and design. 